\title{
UM ESTUDO EMPÍRICO DA APLICAÇÃO DO ÍNDICE EUROPEU DE SATISFAÇÃO DE CLIENTES (ECSI) NO BRASIL
}

\section{AN EMPIRICAL STUDY OF EUROPEAN CUSTOMER SATISFACTION INDEX (ECSI) APPLICATION IN BRAZIL}

RAMON SILVA 느트트

Mestre em Administração pela Faculdade de Estudos Administrativos de Minas Gerais (FEAD-MG) e doutorando em Administração pela

Universidade Presbiteriana Mackenzie (UPM). Coordenador do curso de Administração da Pontifícia Universidade Católica de Minas Gerais (PUC-MG). Avenida Guarim Caetano, 565, Nações - Luz - MG - CEP 35595-000 E-mail: ramonsl@pucminas.br

\section{CID GONÇALVES FILHO}

Doutor em Administração pela Universidade Federal de Minas Gerais (UFMG). Mestrado acadêmico em Ciência da Informação pela Universidade Federal de Minas Gerais (UFMG). Professor e pesquisador da Universidade Fundação Mineira de Educação e Cultura (Fumec).

Rua Sevilha, 250, Vila Castela - Nova Lima - MG - CEP 30400-000 E-mail: cid@consumer.com.br 


\section{RESUMO}

Neste artigo implementou-se o modelo de satisfação ECSI (European Customer Satisfaction Index) em uma das principais empresas brasileiras fornecedoras de Enterprise Resource Planning (ERP). O ECSI analisa o relacionamento da satisfação com seus antecedentes (imagem, expectativa, qualidade e valor) e conseqüentes (reclamação e lealdade). Para esta pesquisa, o modelo original foi modificado, retirando-se o construto reclamação. A pesquisa contou com duas fases: na exploratória foram realizadas entrevistas em profundidade e grupo de foco. $\mathrm{Na}$ fase descritiva foi realizado um e-survey, com 684 casos válidos. As escalas foram adaptadas de outros estudos, exceto para o construto qualidade, em que uma nova escala foi proposta e validada. O tratamento estatístico foi apoiado por técnicas multivariadas, incluindo a Modelagem de Equações Estruturais. Constatou-se que existe uma relação positiva forte entre imagem, qualidade e satisfação na propensão dos clientes à lealdade. Os resultados da pesquisa são discutidos, bem como suas implicações para futuras pesquisas e para a prática gerencial.

\section{PALAVRAS-CHAVE}

Satisfação; Qualidade; Lealdade; Modelagem de equações estruturais; ERP.

\section{ABSTRACT}

This research implemented the ECSI model (European Customer Satisfaction Index), in one of the most important Brazilian organization, that supplies ERP systems. The ECSI analyses the relationship among satisfaction with its antecedents (image, expectative quality and value) and its outcomes (complaint and loyalty). The original model was modified, as the complaint construct was dropped from the model. The methodology was implemented using a two phase design. The exploratory phase was developed applying focus groups and in deep interviews. At the descriptive phase, an e-survey was accomplished, obtaining 684 valid cases. 
The scales were obtained from previous studies, except the quality scale, that was developed specifically for this study. The data was analyzed using structural equation modeling and multivariate techniques. It was observed that there is a strong positive impact on loyalty, which was provided by image, quality and satisfaction. Finally, the results are discussed, as the implications for managers and future research.

\section{KEYWORDS}

Satisfaction; Quality; Loyalty; Structural Equations Modeling, ERP.

\section{INTRODUÇ ÃO}

O ambiente organizacional é hoje caracterizado por transformações muito rápidas. A tecnologia, a mudança no comportamento dos consumidores e o surgimento de novos concorrentes, além de alterações na legislação têm levado as empresas a uma alta competitividade, como conseqüência das mudanças rápidas e descontínuas no seu macroambiente.

Essa realidade está contribuindo para que cada vez se torne mais caro obter novos clientes, fazendo que a manutenção de antigos clientes seja muito atraente (BATESON; HOFFMAN, 200I). Assim, torna-se importante identificar os fatores que levam os clientes a continuar comprando de uma mesma empresa por um período prolongado. Diversos estudos (ANDERSON et al., I994; EDVARDSSON et al., 2000; FORNELL, I992; MARTENSEN et al., 2000) verificaram uma relação positiva entre a satisfação e a propensão do cliente à lealdade. Outros estudos demonstraram ainda as relações entre satisfação, lealdade e desempenho econômico, medidos de diversas formas, como retorno sobre investimento, preço das ações, valor agregado etc. (ANDERSON et al., I994).

Atentos a essas questões, surgiram os índices nacionais de satisfação, que procuraram medir a satisfação dos clientes e a propensão desses à lealdade. Esses índices utilizam a metodologia de indicadores múltiplos para avaliar a satisfação e a propensão à lealdade, que seriam as variáveis latentes. O primeiro deles surgiu em I989, na Suécia. Posteriormente, Alemanha (1992), Estados Unidos (I994), Israel e Taiwan (I995) e Nova Zelândia (I996) também desenvolveram seus índices. Em I996, a Comissão Européia encomendou ao MFQ (Mouvement Français pour la Qualité) um estudo de viabilidade, no sentido de desenvolver um Índice Europeu de Satisfação do Cliente, tomando como base as experiências desenvolvidas em outros países (VILARES; COELHO, I999a). 
Em I998, a EFQM (European Foundation for Quality Management) e a CSI University Network definiram as bases para o lançamento de um projeto piloto do Índice Europeu de Satisfação de Cliente, designado como ECSI (European Customer Satisfaction Index), ao qual aderiram outros doze países europeus.

Partindo das premissas e conclusões desenvolvidas por esse projeto na Europa, o presente artigo examinou empiricamente o modelo estrutural utilizado pelo ECSI no Brasil, mais especificamente em uma empresa brasileira fornecedora de softwares Enterprise Resource Planning (ERP). Essa empresa é uma das principais do seu setor, atuando em todo o território nacional, além de filiais em outros países. Atua no mercado há quase vinte anos, fornecendo ERP (ou softwares de gestão integrada) para empresas de diversos portes e segmentos. Verificaram-se, neste artigo, as relações entre os construtos imagem, expectativa, qualidade de produtos e serviços, valor, satisfação e lealdade de clientes, tendo como referência os trabalhos realizados por Zeithaml et al. (I996), Fornell et al. (I996), Dodds et al., (I99I) e Oliver (I997), além de adaptar escalas para empresas fornecedoras de ERP.

\section{REFERENCIAL TEÓRICO}

\subsection{IMAGEM}

A imagem tem um papel importante na relação entre cliente e fornecedor, já que, conforme Deschamps e Nayak (1996, p. 83), "qualquer consumidor inicia seu processo de compra, implícita ou explicitamente, com uma avaliação da imagem".

Webster Jr. (I994, p. 104) argumenta ainda que

a imagem positiva da marca/nome da instituição é uma das mais poderosas formas de diferenciação de um produto/serviço, pois é virtualmente impossível para um concorrente duplicá-la.

Concordando com essa posição, Gomes e Sapiro (I993) consideram a imagem "um dos ativos mais preciosos que uma organização pode ter".

De acordo com Barich e Kotler (I99I), a percepção que o público-alvo tem de determinada empresa é o reflexo do posicionamento escolhido, isto é, a imagem. Da mesma forma, uma imagem condizente com o posicionamento estratégico torna-se um fator importante de reforço estratégico. 


\subsection{EXPECTATIVAS}

O conceito de expectativas há muito tempo é analisado pelos estudiosos de marketing, especialmente a relação dele com a satisfação de clientes. Entretanto, conforme observam Spreng et al., (I996), não há, na literatura, um consenso claro acerca da definição do conceito de expectativas.

Antes da compra, os clientes têm uma expectativa sobre a qualidade do produto/serviço, com base em necessidades individuais, experiências passadas, recomendações de terceiros e propaganda dos fornecedores. Após comprarem e consumirem o produto/serviço, os clientes comparam a qualidade esperada com aquilo que realmente receberam. Os desempenhos de produtos/serviços que ficarem acima dos seus níveis de serviço desejado serão vistos como de qualidade superior. Se o produto/serviço for analisado dentro de sua zona de tolerância, os clientes tenderão a considerar que ele é adequado. Mas se a qualidade real cai abaixo do nível de serviço adequado e esperado pelos clientes, ocorre uma discrepância - ou lacuna na qualidade - entre o desempenho do fornecedor de serviço e as expectativas do cliente (LOVELOCK; WRIGHT, 200I).

Spreng et al. (1996) tratam as expectativas como "à percepção de probabilidade de ocorrência de determinado evento". Já outros, como Oliver (I980), advogam que o conceito deve ser ampliado e incorporar a avaliação - positiva, negativa ou neutra - do evento esperado. Independentemente dessas diferenças, as duas tendências reconhecem que a expectativa preditiva (isto é, restrita à percepção de probabilidade de ocorrência do evento) é insuficiente para explicar o fenômeno da satisfação.

Assim, diversos autores (EVRARD, I995; OLIVER, I997; SPRENG et al., I996) admitem que as expectativas são apenas um dos elementos do quadro de referência, base pela qual os consumidores avaliam suas experiências.

\subsection{QUALIDADE}

Por ser um tema amplamente abordado por diversos estudiosos, o conceito de qualidade possui diferentes definições. Segundo Horovitz et al. (I994), qualidade pode ser conceituada como "o nível de excelência que a empresa escolheu alcançar para satisfazer sua clientela-alvo e, ao mesmo tempo, a medida em que ela se conforma a esse nível". Esse conceito adota a definição da qualidade baseada no usuário, isto é, na sua percepção. Já Zeithaml (I988) define qualidade percebida como "o julgamento do consumidor sobre a excelência geral ou superioridade do produto ou serviço".

Assim, a qualidade percebida é conceitualmente dividida em dois elementos: qualidade do produto - que consiste na qualidade dos atributos do produto 
físico - e na qualidade dos serviços - que representa a associação interativa entre o cliente e os elementos de serviço, como o comportamento dos funcionários da empresa.

\subsubsection{QUALIDADE DO SOFTWARE}

Com o uso maciço das tecnologias de informação e comunicação em todos os níveis da atividade humana, os problemas de qualidade de software tendem a adquirir a cada dia maior importância.

Diversas metodologias já foram criadas para analisar a qualidade intrínseca de produtos. Mais especificamente no caso de softwares, Pressman (I995) salienta que diversos esforços foram feitos para desenvolver medições precisas da qualidade de software, e essas, às vezes, se frustraram pela natureza subjetiva da atividade. De acordo com esse autor,

é necessário criar formas de medições quantitativas da qualidade de software para análise objetiva. Uma vez que não existe essa coisa de conhecimento absoluto, ninguém deve esperar medir a qualidade de software exatamente, porque cada medição é parcialmente imperfeita.

A norma internacional ISO/IEC 9I26, publicada em I99I, que na versão brasileira de agosto de 1996 recebeu o número NBR I3596, define qualidade de software como "a totalidade de características de um produto de software que lhe confere a capacidade de satisfazer necessidades explícitas e implícitas”.

Necessidades explícitas, nesse caso, são as condições e os objetivos propostos por aqueles que produzem o software. São, portanto, fatores relativos à qualidade do processo de desenvolvimento do produto, e esses fatores são percebidos somente pelas pessoas que trabalharam no seu desenvolvimento. As necessidades implícitas são necessidades subjetivas dos usuários (incluindo operadores, destinatários dos resultados do software e os mantenedores do produto).

Para medir a qualidade de um software surge, porém, um problema. A qualidade de um dispositivo mecânico é freqüentemente medida em relação a tempo médio entre suas falhas, que é uma medida da capacidade de o dispositivo suportar desgaste. Mas o software não se desgasta; portanto, tal método de medição de qualidade não pode ser aproveitado. Nesse sentido, surge a necessidade de avaliar a qualidade do software de acordo com a percepção do usuário, assim como de criar uma ferramenta para mensurar essa percepção. 


\subsubsection{QUALIDADE DOS SERVIÇOS}

Ao contrário da qualidade de bens, que pode ser medida objetivamente, por meio de indicadores como durabilidade e número de defeitos (GARVIN, I992), a qualidade de serviço só pode ser medida parcialmente, em termos objetivos, em razão da natureza mais abstrata do serviço, resultante de sua intangibilidade e demais singularidades (VEIGA, 2000). A qualidade dos serviços está relacionada à habilidade de minimizar as discrepâncias entre as expectativas e percepções de clientes e seus fornecedores.

Segundo Zeithaml (ı988, p. 3), "um serviço é de qualidade somente quando iguala ou supera as expectativas que o cliente tem a respeito dele". Portanto, a avaliação da qualidade do serviço deve ser feita comparando as expectativas de execução com a percepção a respeito do serviço recebido. Esse é o parâmetro que melhor demonstra a satisfação, ou insatisfação, do cliente e, portanto, a qualidade do serviço oferecido. Essa avaliação da satisfação deve ser feita de forma ativa, pelo questionamento e pela comunicação com os clientes.

O modelo conceitual de medição da qualidade do serviço, desenvolvido por Zeithaml et al. (1985), parte do conceito de que a qualidade do serviço se determina pela comparação do serviço esperado com o recebido. A essa diferença os autores chamam "gap 5". Ela é geralmente avaliada com base em cinco dimensões da qualidade do serviço, que são: confiabilidade, capacidade de resposta, segurança, elementos tangíveis e empatia.

O instrumento utilizado para avaliação da qualidade do serviço consiste em um questionário específico para o serviço que se pretende medir. Nesse questionário, refletem-se os atributos para cada dimensão da qualidade do serviço (confiabilidade, garantia etc.). Esses atributos devem ser previamente coletados de diversas fontes, mas, fundamentalmente, dos próprios clientes. O questionário deve medir as expectativas e as percepções de cada atributo. O questionário proporciona informações completas que permitem analisar os aspectos mais valorados pelos clientes e onde são geradas as maiores deficiências. Essa ferramenta, se utilizada adequadamente, pode ser uma valiosa fonte de ajuda para alavancar as melhorias necessárias, de acordo com o requerido pelo cliente, ao mesmo tempo que permite verificar se os esforços e recursos em que a empresa está investindo, com a finalidade de executar seus serviços, estão sendo distribuídos conforme as expectativas e valorações dos clientes.

\subsection{VALOR}

Embora o construto de valor percebido tenha sido identificado por Parasuraman (I997) como uma das mais importantes medidas na busca de vantagem 
competitiva pelas empresas, a literatura a respeito ainda é relativamente restrita (DOMINGUEZ, 2000).

Zeithaml (I988, p. I4) conceitua valor percebido como "a avaliação total do consumidor sobre a utilidade de um produto, baseado em percepções do que é recebido (benefícios) e do que é dado (sacrifícios)". Já Kotler (2000) verifica que o valor percebido é o valor atribuído pelos clientes ao produto ou serviço, baseado na relação entre os benefícios que esse trará, segundo a óptica do consumidor, e os custos percebidos para sua aquisição, comparativamente à concorrência.

De forma sucinta, Gale (1996, p. I6) conceitua o valor como "a qualidade percebida pelo mercado, ajustada pelo preço relativo de seu produto”. Já Woodruff (I997) enxerga o valor como a percepção do cliente em relação às preferências e às avaliações dos atributos do produto, do desempenho desses atributos e das conseqüências originadas pelo uso. Os clientes enxergam o produto como um conjunto de atributos. Assim, quando compram e usam o produto, desenvolvem preferências e desejos por certos atributos, que lhes proporcionam as conseqüências desejadas nas situações de uso (conceito de valor em uso), atendendo a seus objetivos e gerando satisfação com o valor percebido.

\subsection{SATISFAÇÃO DE CLIENTES}

O conceito de satisfação do consumidor vem ocupando uma posição importante no pensamento e na prática do marketing. A satisfação é o resultado mais importante da atividade mercadológica e serve para fazer a ligação entre processos que culminaram em compra e consumo com fenômenos de pós-compra, como atitude de troca, repetição da compra e lealdade à marca.

Conceitualmente, a satisfação é uma conseqüência da compra e uso, resultante da comparação feita pelos consumidores das recompensas e custos da transação em relação às conseqüências antecipadas (CHURCHILL; SUPRENANT, I982). Harvey (I998) concorda com essa definição, salientando que "a satisfação mede quanto os resultados produzidos para o cliente e o processo ao qual ele atravessou para obter esses resultados atendem às suas expectativas".

Oliver (I980), por sua vez, reconhece o papel das expectativas como padrões de comparação, sem os quais a satisfação, como resposta humana, não pode existir. Mas difere do paradigma básico ao considerar a comparação entre expectativas e desempenho como um dos vários processos de comparação que o consumidor realiza ao avaliar um bem ou serviço. Segundo esse modelo, o consumidor pode realizar dois tipos de processos cognitivos (comparações) e psicológicos. O desempenho, afirma esse autor, pode afetar a satisfação diretamente ou, em outras palavras, sem o auxílio de outras influências ou moderadores da satisfação. Mas também é o ponto de partida dos processos de comparação e dos processos psicológicos realizados pelo consumidor. 
Oliver (I980) descreve os processos psicológicos que intervêm na formação de julgamentos de satisfação. Esses processos são menos observáveis, mas são essenciais para compreender o processo de satisfação, por estarem baseados em dissonância cognitiva. Essa dissonância consiste em manifestações de medo e ansiedade que o consumidor pode experimentar no momento de fazer a compra, como resultado de previsões de que o produto não terá o desempenho desejado ou esperado. Segundo o autor, esse temor continua depois da compra, antes do uso e, provavelmente, permanecerá durante o uso do produto.

\subsection{LEALDADE}

O desenvolvimento de relacionamentos fortes e duradouros entre consumidores e fornecedores tem apresentado inúmeros benefícios para ambas as partes. Os benefícios para as empresas têm sido bem documentados na literatura. De maneira geral, clientes leais podem levar ao crescimento dos rendimentos de uma empresa, são mais inclinados a realizar compras adicionais de produtos e serviços e geram novos negócios para a empresa por meio do boca a boca (ZEITHAML et al., I996). Esse ponto de vista é compartilhado por Reichheld (2002), que diz que clientes fiéis costumam recomendar seus fornecedores a outros clientes potenciais, gerando uma fonte adicional de receitas para esses fornecedores.

Anderson et al. (1994) verificaram que um dos principais fatores que levam à lealdade dos clientes é a satisfação destes com o produto e/ou serviço de seus fornecedores. Esses autores apontaram ainda outros benefícios com relação a clientes satisfeitos e leais: redução da sensibilidade ao preço, imunização dos clientes em relação aos esforços da concorrência, custos de transações mais baixos, redução dos custos decorrentes de falhas, custos mais baixos para atrair novos clientes e melhor reputação da empresa.

Existem várias definições para o que se chama de fidelização ou "lealdade do cliente". Para Oliver (I997, p. 6), fidelidade é

um compromisso forte em recomprar ou repatrocinar um produto ou serviço preferido consistentemente no futuro, apesar das influências circunstanciais e tentativas de marketing, que podem acarretar um comportamento de troca.

Já Lovelock e Wright (200I) consideram lealdade

a vontade de um cliente de continuar prestigiando uma empresa durante um período prolongado de tempo, comprando e utilizando seus bens e serviços em uma base repetida e preferivelmente exclusiva, e recomendando voluntariamente os produtos da empresa. 
Oliver (1997), procurando analisar relações entre satisfação e lealdade, verificou que a primeira é um estado temporal pós-uso, decorrente de uma situação de consumo única ou de experiências repetidas, que refletem como um produto ou serviço conseguiu suprir seu propósito. Com base na perspectiva da empresa, a satisfação é entregue ao consumidor. Já a lealdade é um estado de preferência duradouro, obtido por meio de uma postura determinada do cliente para com a empresa. Esse autor conclui afirmando que a satisfação não se transforma em lealdade sem a existência de outros fatores. Dessa forma, um consumidor pode continuar satisfeito, mas não ir além desse estado.

\section{HIPÓTESES DO TRABALHO}

Observando-se a revisão dos autores pertinentes, como Fornell (1996), Oliver (I980), Zeithaml (I988), Anderson e Fornell (2000), dentre outros, e com o objetivo de verificar empiricamente as relações do modelo ECSI em uma empresa fornecedora de ERP, o modelo de pesquisa testado foi alterado, retirando-se o construto reclamação. Desse modo, o modelo proposto apresenta as seguintes relações hipotéticas, conforme a Figura I:

\section{FIGURA I}

\section{ESTRUTURA DO MODELO HIPOTÉTICO PROPOSTO}

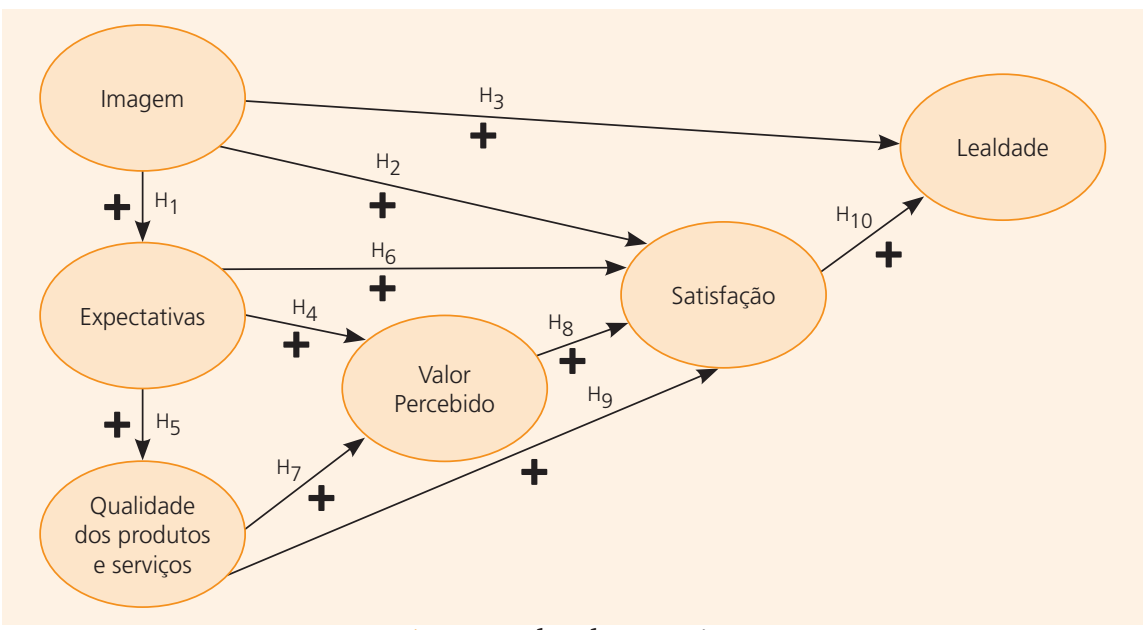

Fonte: Dados da pesquisa.

Assim, as seguintes hipóteses foram propostas:

$\mathrm{H}_{\mathrm{I}}$ - Existe um impacto positivo da imagem percebida na expectativa.

$\mathrm{H}_{2}$ - Existe um impacto positivo da imagem percebida na satisfação de clientes.

$\mathrm{H}_{3}$ - Existe um impacto positivo da imagem percebida na propensão à lealdade. 
$\mathrm{H}_{4}$ - Existe um impacto positivo da expectativa no valor percebido.

$\mathrm{H}_{5}$ - Existe um impacto positivo da expectativa na qualidade.

$\mathrm{H}_{6}$ - Existe um impacto positivo da expectativa na satisfação de clientes.

$\mathrm{H}_{7}$ - Existe um impacto positivo da qualidade no valor percebido.

$\mathrm{H}_{8}$ - Existe um impacto positivo do valor percebido na satisfação de clientes.

$\mathrm{H}_{9}$ - Existe um impacto positivo da qualidade na satisfação de clientes.

$\mathrm{H}_{\text {IO }}$ - Existe um impacto positivo da satisfação de clientes na propensão à lealdade.

\section{MÉTODO}

A pesquisa foi dividida em duas fases. A primeira fase, de caráter exploratório, foi conduzida por meio de entrevistas em profundidade e grupos de foco exploratório. Nessa fase foi feita uma amostragem não probabilística por julgamento. Foram escolhidas dez das vinte organizações que mantêm os mais altos contratos de manutenção vigentes, ligados à matriz da empresa. As entrevistas foram feitas com gerentes de informática e/ou tomadores de decisão.

Os roteiros das entrevistas foram elaborados com base na literatura, privilegiando aspectos histórico-discursivos e descritivos dos fatos referentes ao relacionamento entre essas empresas e a fornecedora de ERP. Objetivou-se listar os itens que deveriam constar no questionário. Após a análise dos dados, um questionário inicial foi desenvolvido, buscando abordar os itens levantados na pesquisa relacionados à qualidade do software. Os itens relacionados aos demais construtos foram obtidos de trabalhos anteriores, realizados por Zeithaml et al. (I996), Fornell et al. (I996), Brady e Cronin (200I) e Oliver (I997). As escalas foram alteradas para onze pontos, visando ao tratamento como variáveis contínuas e possível melhor medição. Esse questionário foi então submetido a um grupo de foco exploratório, composto por doutores, mestres e profissionais da área, que propuseram mudanças no questionário. Em seguida, foi conduzido e analisado um pré-teste com cinqüenta respondentes. Finalmente, os itens de operacionalização dos construtos foram estabelecidos.

A segunda fase seguiu uma estratégia quantitativa por meio de e-survey, utilizado com sucesso por Scornavacca Jr. et al. (200I), os quais ressaltaram que esse método demonstrou-se útil no sentido de alcançar níveis hierárquicos mais elevados nas organizações. Taylor (2000) aponta ainda outras vantagens do e-survey, como possibilidade de trabalhar com grandes amostras, maior velocidade de resposta e redução dos custos operacionais. Esse método permitiu aplicar técnicas de validação de escalas e métricas, o que corroborou os objetivos da pesquisa. Foram analisadas as organizações clientes da empresa que possuíam 
contrato de manutenção vigente na época estudada. Já a unidade de observação da pesquisa foram os gerentes de informática e/ou tomadores de decisão das organizações em análise. Os dados foram coletados por meio de questionários estruturados disponibilizados no site da empresa, além de o mesmo questionário ter sido enviado para a população dos 19.500 clientes, que constavam no banco de dados da empresa. Houve duas levas de questionário, sendo a primeira em 26 de janeiro de 2004, e a segunda, de reforço para aqueles que ainda não haviam respondido, em I6 de fevereiro de 2004 . Os clientes foram incentivados a responder o questionário por meio de sorteio de diversos brindes oferecidos pela empresa.

\section{ANÁLISE dOS DAdOS}

A análise dos dados seguiu metodologicamente uma série de etapas, visando verificar pressupostos e consistência dos dados, verificar confiabilidade e validade das medições e escalas, criando a base para o teste das hipóteses propostas.

\subsection{DADOS AUSENTES E VALORES EXTREMOS (OUTLIERS)}

Da amostra de 862 respondentes foram retirados 54 casos que apresentaram porcentual de dados ausentes significativos, o que comprometeria a análise de dados. Quanto aos valores extremos multivariados, foram retirados I24 casos, uma vez que a distância de Mahalanobis apresentou valores significativos no nível de o,I\%. Dessa forma, a amostra ficou com 684 casos válidos para prosseguir com a análise.

\subsection{AVALIAÇÃO DA DIMENSIONALIDADE DOS CONSTRUTOS POR MEIO DA ANÁLISE FATORIAL}

Neste trabalho, o objetivo da análise fatorial é analisar a presença das dimensões subjacentes presentes nos construtos pesquisados. A existência de correlações significativas entre os itens foi mensurada por meio da estatística denominada de Teste de Esfericidade de Bartlett. O objetivo dessa estatística é testar a hipótese nula de que não há correlação significativa entre os itens, isto é, a matriz de correlação é a matriz-identidade. Se a hipótese nula não for rejeitada, a utilização da análise fatorial pode ser colocada em dúvida. Outra medida de importância fundamental é a medida de adequação da amostra, também conhecida como KMO (Kaiser-Meyer-Olkin). Essa medida compara o valor das correlações 
observadas com os valores das correlações parciais. Pequenos valores de KMO indicam que as correlações entre os pares de variáveis não podem ser explicados por outras variáveis, e que, assim, a análise fatorial não é indicada.

Malhotra (200I) recomenda 0,50 como valor mínimo para a estatística KMO, isto é, valores iguais ou superiores a 0,50 indicam que a análise fatorial é uma técnica apropriada para o conjunto de dados em questão. Segundo Hair et al. (I998), a Medida de Adequação da Amostra (ou KMO) pode ser interpretada da seguinte maneira: $০, 9 \circ$ ou acima, marvelous; ०,8० ou acima, meritorius; $০, 70$ ou acima, middling; o,60 ou acima, mediocre; 0,50 ou acima, miserable; e abaixo de 0,50 , inacceptable. Ressalta-se que a tradução não foi feita ipsis literis, por temer a não-fidelidade ao real sentido com o qual os termos foram utilizados pelos autores.

Outra decisão é quanto ao método de extração e tipo de rotação a serem utilizados na análise fatorial. Como método de extração, usou-se a extração por eixos principais. Segundo Malhotra (200I), esse método é o mais indicado, quando o objetivo principal é verificar a existência de dimensões latentes. Quanto ao método de rotação, foi utilizado o oblíquo, pois esse método parte do pressuposto de uma relação entre os fatores.

Para determinar o número de fatores considerados, Malhotra (200I) apresenta cinco formas. Neste estudo, foram considerados os fatores cujo autovalor superasse i em conjunto com a quantidade de variação explicada.

\section{TABELA I}

KMO E TESTE DE BARTLETT

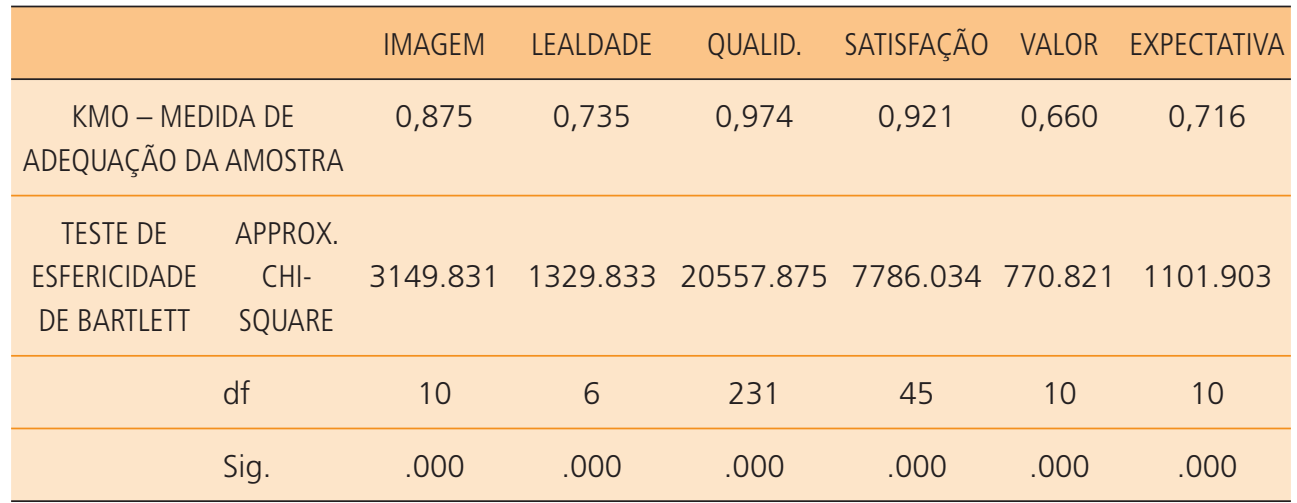

Fonte: Dados de pesquisa, 2004. 
Por meio da Tabela I, observa-se que o valor de KMO para cada construto superou o valor mínimo exigido por Malhotra (200I), ou seja, variou de 0,66 a 0,97. Ademais, observa-se que o Teste de Esfericidade de Bartlett mostrouse significativo para todos os construtos, o que rejeita a hipótese de que não há correlações significativas entre os itens do construto. Essas constatações apóiam empiricamente dois argumentos: (I) o número de respondentes é adequado para a análise fatorial desenvolvida; e (2) há correlações em um nível tal que permite a formação de fatores.

Observou-se ainda que o construto qualidade apresentou quatro fatores: qualidade dos serviços; qualidade dos softwares; qualidade do suporte e qualidade no atendimento, que contabilizaram $73 \%$ da variação observada. Quanto ao construto imagem, verificou-se que é unidimensional, assim como o construto expectativas. Já o construto valor apresentou dois fatores que explicam 47,6\% da variação observada. Entretanto, como será mostrado nas páginas seguintes, o segundo fator foi retirado, por não apresentar um nível de confiabilidade satisfatório.

O construto satisfação apresentou dois fatores: satisfação geral e arrependimento. Esses dois fatores explicam 74,0\% da variação observada. Quanto ao construto lealdade, verificou-se que é unidimensional e que o fator extraído explica $54,86 \%$ da variação observada.

\subsection{ANÁLISE DE CONFIABILIDADE DOS CONSTRUTOS POR MEIO DO ALFA DE CRONBACH}

Para avaliar as propriedades psicométricas das escalas no presente estudo, foi calculado o coeficiente alfa de Cronbach, citado por Malhotra (200I). Segundo esse autor, o coeficiente alfa é a média de todos os coeficientes possíveis resultantes das diferentes divisões da escala em duas metades.

O alfa de Cronbach pode variar de o a I. Dessa forma, quanto mais próximo de I, maior é a confiabilidade da escala. Neste estudo, escalas cujo coeficiente alfa de Cronbach exceder o valor de o,6 serão consideradas fidedignas, como recomenda Malhotra (200I).

Além do valor do coeficiente, Hair et al. (1998) sugerem a análise do grau de correlação que cada item da escala exibe com toda a escala e com cada item individualmente. Para os autores, o pesquisador pode considerar a seguinte regra prática: a correlação entre um determinado item e o total da escala deve ser superior a 0,50 , e a correlação entre um determinado item e outro deve ser superior a 0,30 . 


\section{TABELA 2}

CONSISTÉNCIA INTERNA PELO ALFA DE CRONBACH

\begin{tabular}{lcc}
\hline \multirow{2}{*}{ CONSTRUTOS } & DIMENSÕES & ALFA DE CRONBACH \\
\hline Qualidade & Fator 1 & 0,9714 \\
\cline { 2 - 3 } & Fator 2 & 0,8392 \\
\cline { 2 - 3 } & Fator 3 & 0,9420 \\
\hline Imagem & Fator 4 & 0,9484 \\
\hline Valor & Apenas um fator & 0,9229 \\
\cline { 2 - 3 } & Fator 1 & 0,7225 \\
\hline Satisfação & Fator 2 & 0,4250 \\
\hline Expectativas & Fator 1 & 0,9544 \\
\hline Lealdade & Fator 2 & 0,8638 \\
\hline & Apenas um fator & 0,7828 \\
\hline
\end{tabular}

Fonte: Dados de pesquisa, 2004.

Por meio da Tabela 2, observa-se que apenas o Fator 2 do construto valor não apresentou um coeficiente alfa superior a $\circ, 6 \circ$. Os demais valores superam o limite variando de 0,7225 a 0,9714 . Esses resultados sustentam o argumento de que as escalas desenvolvidas são fidedignas, principalmente aquelas desenvolvidas especificamente para este trabalho, que seriam as escalas referentes à qualidade, já que as escalas utilizadas nos demais construtos foram adaptadas de outros trabalhos. Ressalta-se ainda que o Fator 2 do construto valor foi descartado, por mostrar-se com a confiabilidade abaixo do limite mínimo estabelecido neste estudo.

\subsection{ANÁLISE DE CONFIABILIDADE DOS CONSTRUTOS POR MEIO DA VARIÂNCIA EXTRAÍDA}

Não obstante seu uso intenso na avaliação de confiabilidade de escalas, Hair et al. (I998) apontam limitações no coeficiente alfa de Cronbach. Para os autores, no cálculo da confiabilidade por meio do alfa de Cronbach não são conside- 
rados os erros nos indicadores. Dessa forma, os autores encorajam a utilização da confiabilidade composta e da variância extraída por meio de análise fatorial confirmatória. Esses autores consideram como satisfatórios valores iguais ou superiores a 0,50 , tanto para a confiabilidade composta como para a variância extraída, embora afirmem que valores menores podem ser aceitos, dependendo das características da pesquisa, a critério do pesquisador.

Excetuando-se o construto satisfação, que apresentou valores próximos do aceitável, todos os demais construtos atingiram valores acima de 0,50 . Nesse sentido, verifica-se que todas as escalas foram consideradas aceitáveis. Vale ressaltar que a escala do construto qualidade foi desenvolvida por este estudo e, não obstante, foi a que apresentou os mais altos índices, sendo o,968 para a confiabilidade composta e 0,885 para variância extraída.

\section{TABELA 3}

ANÁLISE DE CONFIABILIDADE COMPOSTA E VARIÂNCIA EXTRAÍDA

\begin{tabular}{ccc}
\hline CONSTRUTO & CONFIABILIDADE COMPOSTA & VARIÂNCIA EXTRAÍDA \\
\hline Qualidade & 0,968 & 0,885 \\
\hline Imagem & 0,944 & 0,770 \\
\hline Lealdade & 0,865 & 0,686 \\
\hline Expectativas & 0,897 & 0,687 \\
\hline Satisfação & 0,472 & 0,312 \\
\hline Valor & 0,75 & 0,51 \\
\hline
\end{tabular}

Fonte: Dados de pesquisa, 2004 .

\subsection{ANÁLISE DA VALIDADE DISCRIMINANTE}

A validade discriminante foi realizada seguindo o procedimento recomendando por Bagozzi et al. (I99I). Para isso, testa-se a correlação de construto par a par, por meio da modelagem por equações estruturais. A lógica desse tipo de análise consiste na comparação entre dois modelos testados para cada par de construtos; um modelo no qual a covariância é forçada em I, e outro modelo no qual a covariância é deixada livre. Calcula-se a diferença entre as estatísticas Qui-quadrado obtidas pelos dois modelos. Se essa diferença ultrapassar 3,84 (Qui-quadrado crítico a I grau de liberdade), constata-se que os construtos são diferentes, ou seja, são estatisticamente diferentes. 
De acordo com a análise dos dados, verificou-se que a diferença em todos os pares de construtos foi significativa, o que demonstra que a validade discriminante foi atestada, uma vez que o valor referente à diferença entre Qi-quadrados apresentados pelos construtos superou, em todos os pares comparados, o valor de 3,84. Constata-se, com isso, que a escala apresenta realmente a medida para a qual foi criada para medir, demonstrando sua validade.

\subsection{FASE EXPLICATIVA}

Realizada a análise de validação do instrumento de pesquisa, parte-se então para a aplicação da técnica de equações estruturais, sendo essa uma técnica avançada de tratamento e análise estatística, conforme salienta Gosling e Gonçalves (2003).

Equações estruturais têm sido utilizadas em diversos campos de estudo. A razão para esse fato, de acordo com Gonçalves Filho (200I), se baseia em dois aspectos: (I) provê um método para lidar com múltiplos relacionamentos simultaneamente, enquanto provê eficiência estatística; (2) sua habilidade em avaliar os relacionamentos de forma abrangente e prover uma transição da análise exploratória para a confirmatória.

Para o teste do modelo hipotético, optou-se pelo processo de estimação direta, utilizando-se como matriz de entrada a matriz de covariância, conforme aconselha Hair et al. (I998). O método de estimação escolhido para esta pesquisa foi ML, que, segundo Hair et al. (I998), é um método de estimação adequado quando os dados são moderadamente não normais, levando-se em conta o tamanho possível da amostra. Conforme Gonçalves Filho (200I), o método máxima verossimilhança (Maximum Likelihood, M.L., no original, em inglês) tem sido a abordagem mais comumente utilizada na modelagem de equações estruturais.

As relações estruturais para validação de hipóteses e modelos foram realizadas utilizando-se o software AMOS 4.0, da SPSS. O modelo é apresentado na Figura 2. 


\section{FIGURA 2}

\section{MODELO DA PESQUISA - PESOS PADRONIZADOS}

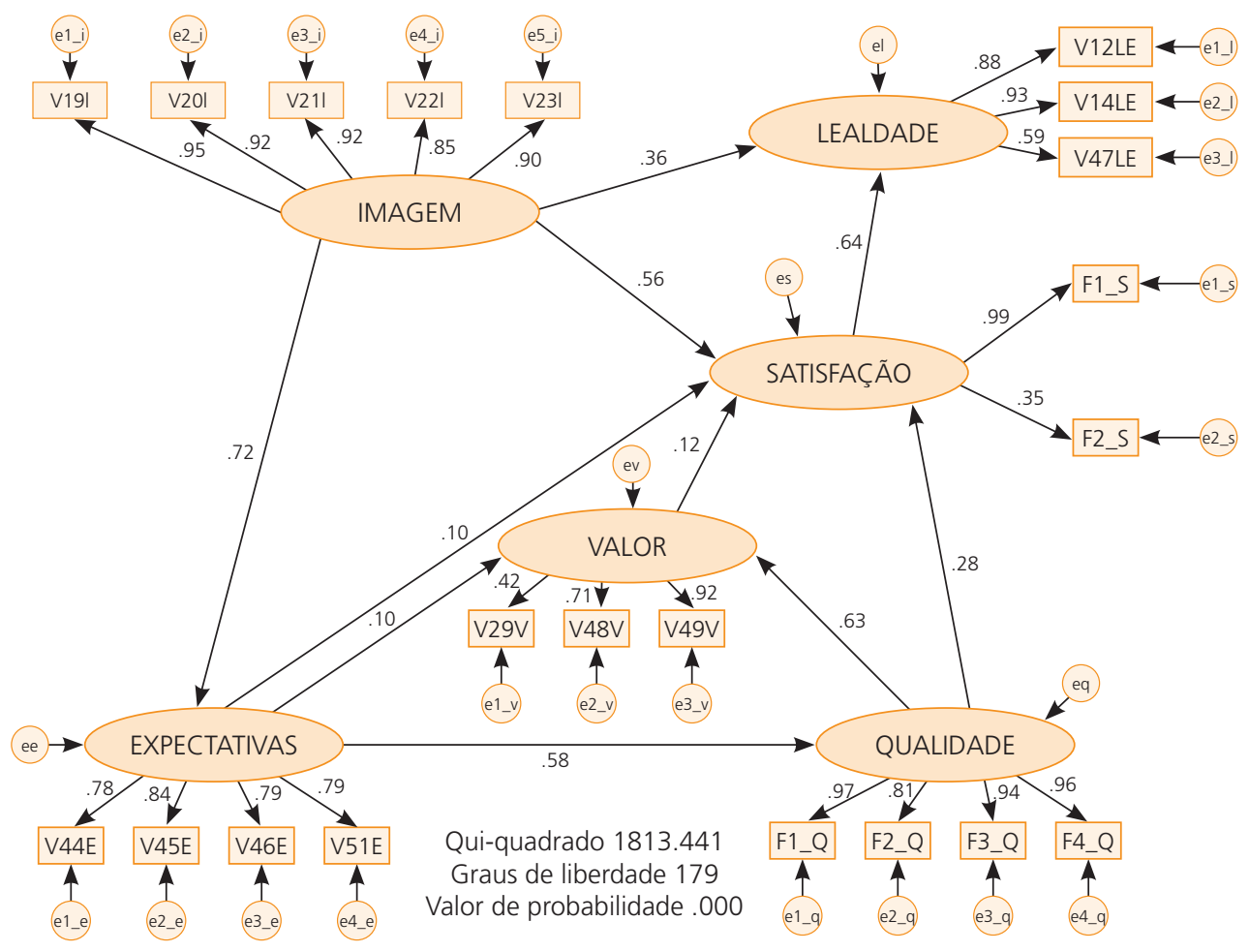

Fonte: Dados de pesquisa, 2004 .

TABELA 4

TESTES DAS HIPÓTESES REFERENTES

AS LIGAÇÕES ENTRE CONSTRUTOS - ML

\begin{tabular}{|c|c|c|c|c|c|c|c|}
\hline & & & $\begin{array}{r}\text { COEFICIEN } \\
\text { PADRON }\end{array}$ & $\begin{array}{l}\text { ES NÃO } \\
\text { ZADOS }\end{array}$ & $\begin{array}{c}\text { COEFICIENTES } \\
\text { PADRONIZADOS }\end{array}$ & & \\
\hline RELACIONA & MEN & ANALISADO & ESTIMADO & $\begin{array}{c}\text { ERRO } \\
\text { PADRÃO }\end{array}$ & $\beta$ & VALOR T & VALOR P \\
\hline Expectativas & $\leftarrow$ & Imagem & 0,540 & 0,027 & 0,72 & 20,055 & 0,000 \\
\hline Qualidade & $\leftarrow$ & Expectativas & 0,752 & 0,049 & 0,58 & 15,237 & 0,000 \\
\hline Valor & $\leftarrow$ & Qualidade & 0,627 & 0,042 & 0,63 & 14,979 & 0,000 \\
\hline
\end{tabular}




\section{TABELA 4 (CONCLUSÃO)}

TESTES DAS HIPÓTESES REFERENTES

AS LIGAÇÕES ENTRE CONSTRUTOS - ML

\begin{tabular}{|c|c|c|c|c|c|c|c|}
\hline & & & $\begin{array}{r}\text { COEFICIEN } \\
\text { PADRON }\end{array}$ & $\begin{array}{l}\text { IES NÃO } \\
\text { ZADOS }\end{array}$ & $\begin{array}{l}\text { COEFICIENTES } \\
\text { PADRONIZADOS }\end{array}$ & & \\
\hline RELACION & MEN & ANALISADO & ESTIMADO & $\begin{array}{c}\text { ERRO } \\
\text { PADRÃOO }\end{array}$ & $\beta$ & VALORT & VALOR P \\
\hline Satisfação & $\leftarrow$ & Expectativas & 0,122 & 0,045 & 0,10 & 2,718 & 0,007 \\
\hline Satisfação & $\leftarrow$ & Imagem & 0,498 & 0,029 & 0,56 & 17,484 & 0,000 \\
\hline Satisfação & $\leftarrow$ & Valor & 0,112 & 0,029 & 0,12 & 3,827 & 0,000 \\
\hline Satisfação & $\leftarrow$ & Qualidade & 0,258 & 0,030 & 0,28 & 8,575 & 0,000 \\
\hline Lealdade & $\leftarrow$ & Imagem & 0,325 & 0,038 & 0,36 & 8,508 & 0,000 \\
\hline Lealdade & - & Satisfação & 0,656 & 0,048 & 0,64 & 13,650 & 0,000 \\
\hline
\end{tabular}

Fonte: Dados de pesquisa, 2004 .

Por meio da Tabela 4, observa-se que nenhuma hipótese foi rejeitada estatisticamente, no nível de $5 \%$, isto é, todas as hipóteses propostas foram confirmadas. Verifica-se ainda que o valor crítico de t, no nível de 5\%, é superior a I,96, o que mostra que os pesos nos caminhos são estatisticamente significativos.

Constata-se também um forte impacto da imagem nas expectativas $(\beta=0,72)$. A qualidade é influenciada pelas expectativas $(\beta=0,58)$ e tem importante influência no valor percebido $(\beta=0,63)$. Além disso, o $\beta=0,656$ demonstra a importância da satisfação dos clientes para se obter a lealdade deles, confirmando outros estudos como o de Fornell et al. (1996) e Veiga (2000).

Os indicadores de ajuste do modelo são apresentados na Tabela 5.

TABELA 5

INDICADORES DE AJUSTE DO MODELO

\begin{tabular}{cccccccc}
\hline & & QUI-QUADRADO / & & & & \\
QUI-QUADRADO & GRAUS DE LIBERDADE & GRAUS DE LIBERDADE & RMR & GFI & CFI & RMSEA \\
\hline 1813,441 & 179 & 10,131 & 0,726 & 0,826 & 0,865 & 0,116 \\
\hline
\end{tabular}

Fonte: Dados de pesquisa, 2004. 
Por meio da Tabela 5, constata-se que o ajuste absoluto não existiu, pois a relação Qui-quadrado/Graus de Liberdade mostrou-se acima de 6, GFI e CFI abaixo de 0,9 e, finalmente, o RMSEA é maior que zero.

Constata-se, com isso, que o modelo ECSI não demonstrou um ajuste global para empresas fornecedoras de ERP. Isso demonstra que ajustes são necessários e que novos estudos deveriam ser realizados, na busca de atingir um modelo que seja adequado às especificidades desse setor.

\subsection{CONSIDERAÇÕES GERAIS SOBRE OS RESULTADOS}

Observou-se que as escalas apresentaram coeficiente de confiabilidade, medido pelo alfa de Cronbach, acima de o,70, o que reflete uma fidedignidade aceitável. Essa consideração é confirmada pela análise da confiabilidade composta e variância extraída, que mostra que todos os construtos atingiram valores aceitáveis. Por meio da análise discriminante, foi constatada ainda a validade da escala, pois todos os pares de construtos obtiveram valores acima do limite aceitável.

A análise do modelo leva-nos a observar que a qualidade $(\beta=0,63)$ tem forte impacto sobre o valor. Além disso, a satisfação é influenciada positivamente pelo valor, pelas expectativas, pela qualidade e pela imagem, estas últimas com maior peso, respectivamente $(\beta=0,28$ e $\beta=0,56)$. Verificou-se também que a imagem $(\beta=0,36)$ e a satisfação $(0,64)$ têm impacto positivo na lealdade dos clientes. Essa constatação encontra sustentação em outras pesquisas realizadas, tais como de Fornell et al. (1996), Gonçalves Filho (200I) e Veiga (2000). Nesse sentido, empresas do setor de software devem investir seus esforços em imagem e qualidade para obterem a satisfação de seus clientes, aumentando assim a propensão deles à lealdade.

Pelo uso de equações estruturais, constatou-se que o modelo não apresentou um ajuste global adequado. Nesse sentido, o modelo precisa ser mais bem adequado à realidade brasileira, mais especificamente ao segmento de software, que possui características distintas.

\section{CONCLUSÕES}

O tamanho da amostra, a abrangência da pesquisa e os bons resultados obtidos na validação das escalas utilizadas para medir as variáveis em análise permitiram o teste do modelo proposto por meio da técnica de modelagem em equações estruturais. Esse teste do modelo serviu para a avaliação das hipóteses elaboradas no trabalho. 
A aplicação, no Brasil, de um estudo como este, já realizado em outros países, constitui uma oportunidade única para a avaliação da generalidade de um modelo teórico, bem como para a certificação de validade dos instrumentos de pesquisa utilizados. O exame do modelo ECSI em empresas fornecedoras de ERP possibilitou a conclusão de que é necessário considerar as especificidades desse setor. Porém, o desenvolvimento de uma escala de mensuração da qualidade de software, com base nos trabalhos realizados por Zeithaml et al. (I996), foi o subproduto mais relevante deste trabalho.

Quanto às hipóteses inicialmente apresentadas, todas elas foram apoiadas pelos testes, confirmando a imagem, a expectativa, o valor percebido e a qualidade como antecedente da satisfação e tendo a lealdade como conseqüente.

\subsection{LIMITAÇÕES E RECOMENDAÇÕES PARA PESQUISAS FUTURAS}

A generalização dos resultados deste estudo para todas as empresas de software fica comprometida, uma vez que o produto estudado, o ERP, apresenta características específicas. Sugere-se, portanto, a ampliação desta pesquisa, para que se possam contemplar empresas que forneçam outros tipos de softwares.

A seleção da amostra de clientes em apenas uma empresa (mesmo tendo abrangência nacional e sendo uma das principais organizações do mercado em que atua) também pode ser considerada uma limitação do trabalho.

Uma sugestão para futuras pesquisas é a aplicação do modelo ECSI completo, de maneira que contemple também o construto reclamações, no sentido de analisar o impacto desse na lealdade e como é influenciado pela satisfação. Seria interessante que isso fosse realizado em setores da economia já pesquisados na Europa, o que levaria a comparações de índices entre países.

\section{REFERÊNCIAS}

ANDERSON, E. W. et al. Customer satisfaction, market share and profitability: findings from Swedem. Journal of Marketing, Chicago, v. 55, p. 53-66, April I994.

ANDERSON, E. W.; FORNELL, C. Foundations of the American Customer Satisfaction Index. Total Quality Management, p. 869-882. 2000.

BAGOZZI, R. P. et al. Assessing construct validity in organizational research. Administrative Science Quaterly, v. 36, n. 3, p. 42I-458, Sept. I99I.

BARICH, H.; KOTLER, P. A framework for marketing image management. Sloan Management Review, v. 32, n. 2, I99I.

BATESON, J. E. G.; HOFFMAN, K. D. Marketing de serviços. 4. ed. Porto Alegre: Bookman, 200 . 
- UM ESTUDO EMPÍRICO DA APLICAÇÃO DO ÍNDICE EUROPEU DE SATISFAÇÃO DE CLIENTES (ECSI) NO BRASIL• RAMON SILVA LEITE • CID GONÇALVES FILHO

BRADY M.; CRONIN, K. J. J. Some new thoughts on conceptualizing perceived service quality: a hierarchical approach. Journal of Marketing, Chicago, v. 65, n. 3, p. 34-49, Jul. 200 I.

CHURCHILL, G. A.; SUPRENANT, C. An investigation into the determinants of customer satisfaction. Journal of Marketing Research, v. I9, p. 491-504, Nov. I982.

DESCHAMPS, J. P.; NAYAK, P. R. Produtos irresistíveis - como operacionalizar um fluxo perfeito de produtos do produtor ao consumidor. São Paulo: Makron Books, I996.

DODDS, W. B. et al. The effects of price, brand and store information on buyers product evaluations. Journal of Marketing Research, v. 28, p. 307-319, Ago. I991.

DOMINGUEZ, S. V. O valor percebido como elemento estratégico para obter a lealdade dos clientes. Caderno de Pesquisas em Administração, São Paulo, v. 7, n. 4, out./dez. 2000.

EDVARDSSON, B. et al. The effects of satisfaction and loyalty on profits and growth - Products versus services. Total Quality Management \& Business Excellence, v. II, n. 7, p. 9I7-927, Sept. 2000.

EVRARD, Y. A satisfação dos consumidores: situação das pesquisas. Porto Alegre: PPGA/UFRGS, I995. (Mimeogr.)

FORNELL, C. et al. The American Customer Satisfaction Index: nature, purpose and findings. Journal of Marketing, Chicago, v. 6o, p. 7-18, out. I996.

FORNELL, C. A national customer satisfaction barometer: the Swedish experience. Journal of Marketing, Chicago, v. 56, p. 6-21, Jan.1992.

Customer satisfaction and shareholder value. In: FOURTH WORLD CONGRESS FOR TOTAL QUALITY MANAGEMENT, Sheffield, 28-30 de jun. I999.

GALE, B. T. Gerenciando o valor do cliente: criando qualidade e serviços que os clientes podem ver. São Paulo: Pioneira, I996.

GARVIN, D. A. Gerenciando a qualidade: a visão estratégica e competitiva. Rio de Janeiro: Qualitymark, I992.

GOMES, M. T.; SAPIRO, A. Imagem corporativa - uma vantagem competitiva sustentável. Revista de Administração de Empresas, São Paulo, v. 33, n. 6, I993.

GONÇALVES FILHO, C. O impacto da gestão do conhecimento de marketing na inovação e vantagem competitiva de novos produtos. Belo Horizonte, 200I. Tese (Doutorado) - Faculdade de Ciências Econômicas, Universidade Federal de Minas Gerais.

GOSLING, M.; GONÇALVES, C. A. Modelagem por equações estruturais: conceitos e aplicações. FACES Revista de Administração, Belo Horizonte, v. 2, n. 2, 2003.

HAIR J. F. et al. Multivariate Data Analysis. New Jersey: Prentice Hall, I998.

HARVEY, J. Service quality: a tutorial. Journal of Operations Management, Amsterdam, v. I6, n. 5, p. 583-897, Oct. I998.

HICKS, D. A. The manager's guide to supply chain and logistics problem-solving tools and techniques. IIIE Solutions, v. 29, Iss.IO, p. 24-29, I997.

HOROVITZ, J. L. et al. Qualidade de serviço: a batalha pela conquista do cliente. São Paulo: Nobel, I994.

ISO/IEC DIS I4598-5. Information Technology Evaluation of software product. Part 5: Process for evaluators. Disponível em: <http://www.cse.dcu.ie/essiscope/sm4/I4598-5.html>. Acesso em: 23 set. 2003.

KOTLER, P. Administração de Marketing. São Paulo: Prentice-Hall, 2000.

LAUDON, K. C.; LAUDON, J. P. Management information systems. 6. ed. Upper Saddle River: Prentice Hall, 2000. 
LOVELOCK, C.; WRIGHT, L. Serviços, marketing e gestão. São Paulo: Saraiva, 200I. MALHOTRA, N. K. Pesquisa de Marketing: uma orientação aplicada. Porto Alegre: Bookman, 200 I. MARTENSEN, A. et al. The drivers of customer satisfaction and loyalty: cros-industry findings from Denmark. Total Quality Management, v. II, n. 5, 2000.

OLIVER, R. L. A cognitive model of the antecedents and consequences of satisfaction decisions. Journal of Marketing Research, v. I7, p. 460-469, Nov. I980.

Measurement and evaluation of satisfaction processes in retail settings. Journal of Retailing,

v. 57 , p. $25-48$, I98I.

Satisfaction: a behavioral perspective on the consumer. New York: McGraw-Hill, I997.

PARASURAMAN, A. et al. A conceptual model of service quality and its implications for future research. Journal of Marketing, n. 49, p. 4I-50, Jul. I985.

PARASURAMAN, A. Reflections on gaining competitive advantage through customer value. Journal of the Academy of Marketing Science, n. 25, p. I54-I6I, I997.

PRESSMAN, R. S. Engenharia de software. São Paulo: Pearson Education do Brasil, I995.

REICHHELD F. F. Princípios da lealdade. Rio de Janeiro: Campus, 2002.

SCORNAVACCA JUNIOR, E. et al. E-survey: concepção e implementação de um sistema de survey pela internet. In: XXV EnANPAD. Anais... Campinas, 200I.

SPRENG, R. A. et al. A Reexamination of the Determinants of Consumer Satisfaction. Journal of Marketing, v. 60, p. 15-32, Jul. 1996.

TAYLOR, H. Does Internet research work? Comparing online survey results with telephone survey. International Journal of the Market Research Society, v. 42, n. I, p. 51-63, 2000.

VEIGA, R. T. Um exame empírico do modelo de conseqüencias comportamentais da qualidade de serviços. Belo Horizonte, 2000. Tese (Doutorado) - Faculdade de Ciências Econômicas, Universidade Federal de Minas Gerais.

VILARES, M. J.; COELHO, P. S. Índice nacional de satisfação de clientes. In: $24^{\circ}$ COLÓQUIO DA $\mathrm{APQ}$, Lisboa, I999a.

The employee-customer satisfaction chain. In: CONFERENCE OF CUSTOMER SATIS-

FACTION: THEORY AND MEASUREMENT, Vienna, May, 20 e 2I, I999b.

WEBSTER JUNIOR, F. E. Marketing driven management. New York: John Wiley \& Sons, I994.

WOODRUFF, R. Customer Value: the next source for competitive advantage. Journal of the Academy of Marketing Science, Greenvale, v. 25, n. 2, p. I39-153, I997.

ZEITHAML, V. A. Consumer perception of price, quality and value: a means-end model and synthesis of evidence. Journal of Marketing, Chicago, v. 52, Jul. I988.

ZEITHAML, V. A. et al. Problems and strategies in services marketing. Journal of Marketing, Chicago, v. 49, p. 33-46, Abril I985. The behavioral consequences of service quality. Journal of Marketing, Chicago, v. 6o, April I996.

\section{TRAMITAÇ ÃO}

Recebido em 26/4/2006

Aprovado em 20/7/2006 
Copyright of Revista de Administração Mackenzie is the property of Universidade Presbiteriana Mackenzie, RAM-Revista de Administracao Mackenzie and its content may not be copied or emailed to multiple sites or posted to a listserv without the copyright holder's express written permission. However, users may print, download, or email articles for individual use. 